\title{
Thin Layer Chromatographic Analysis of Annatto Extracts Obtained Using Supercritical Fluid
}

\author{
Carolina L. C. Albuquerque ${ }^{1}$, Ádina L. Santana², M. Angela A. Meireles ${ }^{2, *}$ \\ ${ }^{1}$ DTA (Food Technology Department)/CTDR (Technological and Regional Development Center)/UFPB (Federal University of Paraíba), \\ Av. Escoteiros, S/N, João Pessoa-PB, Brazil \\ ${ }^{2}$ LASEFI/DEA/FEA (School of Food Engineering), UNICAMP (University of Campinas), R. Monteiro Lobato, Campinas-SP, Brazil
}

\begin{abstract}
The goal of this paper was to qualitatively identify the chemical composition of annatto extracts obtained by supercritical fluid extraction (SFE) using thin layer chromatography (TLC) in terms of volatile oil compounds, compounds with antioxidant activity and phenolic compounds. The mobile phase that achieved the best separation of the studied compounds of interest presented in the extracts was used. The $\gamma$-tocotrienol and $\delta$-tocotrienol antioxidants as well as squalene and $\beta$-sitosterol were identified by comparison of their $\mathrm{Rf}$ values to the same constituents present in the $30 \%$ Tocomin commercial sample. The chemical profile of extracts varied as a function of extraction time. The intensity of antioxidants, phenolic compounds and volatile oil compounds was reduced, while the intensity of carotenes (bixin) was increased.
\end{abstract}

Keywords Annatto, Bixin, Supercritical fluid extraction, Tocotrienols

\section{Introduction}

Bixin obtained from Bixa orellana L. (annatto) is the most important natural food grade colorant next to saffron in the global market with a current consumption of 10,650 tons [1]. Brazil is one of the major producers and exporters of annatto seeds and extracts. These products have been used to produce various dairy and fat-based products, such as butter, margarine, cheese, baked foods and snack foods, and they have been used in pharmaceutical products, leather dyes and cosmetics [2].

Several carotenoids can be obtained from annatto seeds, mainly bixin and norbixin, and other carotenoids, including $\beta$-carotene, cryptoxanthin, lutein, zeaxanthin and methyl bixin, can be obtained but in a lesser quantity [3].

In addition to the presence of carotenoids, annatto seed extracts also contain phenolic compounds, namely hypoletin as the major compound and a caffeoyl acid derivative as the minor derivative. The levels of phenolic compounds in annatto seed extracts are 20-30 times lower than the bixin contents [4].

High or low extract yields can be obtained for annatto depending on the used extraction method as follows: indirect extraction with solvents; direct extraction using aqueous alkali solutions [5]; supercritical fluid extraction, SFE [6]; pressurized liquid extraction, PLE; and low-pressure solvent extraction, LPSE (that is, atmospheric pressure solvent

* Corresponding author:

maameireles@gmail.com (M. Angela A. Meireles)

Published online at http://journal.sapub.org/fph

Copyright (C) 2015 Scientific \& Academic Publishing. All Rights Reserved extraction) [7].

Global yield isotherms (GYIs) are used to select the operational conditions of temperature and pressure based on the extraction yield and the chemical composition of the extract. After this selection, the overall extraction curve (OEC) must be determined because it provides information about the kinetic behavior of the SFE process [8].

Both carotenoids and phenolic compounds are considered to promote human health because they are responsible for critical biological functions [9].

High-pressure technologies involving sub- and supercritical fluids offer the possibility to obtain new products with special characteristics or to design new processes, which are environmentally friendly and sustainable [10]. Supercritical fluid extraction with carbon dioxide is important in natural compound extraction, especially temperature unstable natural compounds [11].

Determination and separation of various constituents can be performed by a variety of analytical techniques including gas chromatography (GC), high-performance liquid chromatography (HPLC), thin-layer chromatography (TLC), capillary electrophoresis (CE) and nuclear magnetic resonance (NMR) spectroscopy.

Thin-layer chromatography (TLC) and its refined version, high-performance thin-layer chromatography (HPTLC), is an established method of lipid analysis whereby normal phase TLC is traditionally used [12].

With its advantages of simplicity, economy, easy operation and required for only small amounts of solvent, TLC is used widely in various fields to separate or purify mixtures of chemical and biological compounds [13].

The mobile phase is the transport medium for the 
compounds to be separated as it migrates through the stationary phase by capillary forces. The type of the mobile phase influences the separation of compounds. The polarity and volatility of the mobile phase as well as the solubility of the components to be separated have to be considered [14].

Recently, TLC has been used to qualitatively analyze food samples, such as commercial drinks [15], honey bee [16], pitanga [17], jambú [18], shrimp [19] and cherry [20]. Nevertheless, there is a scarcity of recent available information on the chemical profile of annatto seed analyzed by chromatography. TLC has been applied to qualitatively identify the total carotenoids in annatto, carrot, corn and tomato extracts [21]. Static headspace gas chromatography (HSGC) has been applied to quantify residual solvents in annatto extracts [22]. HPLC and mass spectrometry have been used to identify and quantify the carotenoids and phenolic compounds in annatto seeds [9], HPLC analysis has been used to identify the $\gamma$-tocotrienol and $\delta$-tocotrienol compounds in annatto oil obtained by supercritical fluid extraction [23].

The TLC separation process consists mainly of the adsorption principle. The adsorption of the solute occurs in the interface between the solid and the mobile phase due to the presence of active groups on its surface. The desorption of the solute implies its return to the mobile phase due to the volatility or solubility in the mobile phase [24].

The TLC method allows the rapid identification of the food coloring present in samples by comparison of their $\mathrm{Rf}$ (ratio of fronts or retention factor) values and the colors of the extract samples compared to standards [15].

The Rf value depends on the difference of solubilities of the analyte between the stationary and mobile phases [24]. The $\mathrm{Rf}$ value is defined as the ratio of the distance travelled by a component to the distance travelled by the solvent front. The Rf value indicates the solubility of the spot components in the TLC plate [25].

This study qualitatively evaluated the performance of TLC in determining the composition changes in the chemical profile of annatto seed extracts obtained by SFE through the acquisition of global yield isotherms (GYIs) and the overall extraction curves (OECs).

Additionally, standards were used to assist the identification of compounds present in the extracts when known compounds were present in the mixture. The identification of known compounds was performed by the comparison of respective standards through $\mathrm{Rf}$ calculation.

\section{Material and Methods}

\subsection{Raw Material}

Annatto variety Piave was obtained from IAC (Agronomic Institute of Campinas) at the Department of Agriculture and Supply of São Paulo, Brazil.

Immediately after acquisition, the sample was identified and stored at $225 \mathrm{~K}$ in the dark until further analysis.

\subsection{Obtainment of Extracts}

Global yield isotherms (GYIs) and overall extraction curves (OECs) from whole annatto seeds were determined at 313 and $333 \mathrm{~K}$ as well as 20,31 and $40 \mathrm{MPa}$ in a $290 \mathrm{ml}$ extraction vessel using supercritical carbon dioxide [6].

The extract was collected in a $100 \mathrm{ml}$ amber glass vial immersed in an ice bath at ambient pressure. After the extraction, the glass vial remained in a desiccator for ten minutes to allow for the complete elimination of $\mathrm{CO}_{2}$. The total extract mass was determined as the sum of the extract obtained during the extraction and the recovered extract in the cleaning process.

The cleaning process consisted of recovering extract from the tubing line leading out of the extraction vessel with acetone. The glass vials (used in both the extraction and cleaning processes) were flushed with $\mathrm{N}_{2}$ and stored at $255 \mathrm{~K}$ until analysis.

\subsection{Chemicals}

\subsubsection{Reagents}

Ethyl acetate, acetone and n-hexane (99.8\%) were obtained from Merck (Darmstadt, Germany). Glacial acetic acid and concentrated sulfuric acid $(99.8 \%)$ were purchased from Synth (Diadema, Brazil). Formic acid, petroleum ether and methanol were acquired from Ecibra (Santo Amaro, Brazil).

The p-anisaldehyde, 2, 2-diphenyl-1-picrylhydrazyl and 2-aminoethyl diphenylborinate $(99.8 \%)$ reagents were purchased from Sigma-Aldrich (Darmstadt, Germany). Carotech (Perak, Malaysia) donated Tocomin ${ }^{\circledR}(30 \%)$.

Due to the difficulty in finding suppliers and the high cost of commercial forms of tocotrienols, the $30 \%$ Tocomin ${ }^{\circledR}$ commercial sample was used to identify the isomers of tocotrienols on the TLC plates.

The commercial sample was an oil $(30 \%$ tocotrienol suspension), and it was comprised predominantly of $\alpha-, \beta-, \gamma-$, and $\delta$-tocotrienol as well as $\alpha$-tocopherol, squalene and other minor constituents, such as sterols ( $\beta$-sistosterol) and carotenoids ( $\beta$-carotene). The constituents were extracted and concentrated by a molecular distillation process from crude palm (Elaeis guineensis) oil, which was provided by Carotech Inc. Berhad (Malaysia).

\subsubsection{Standards}

$\beta$-sistosterol (95\%), $\alpha$-tocopherol (97\%) and stigmasterol $(95 \%), \beta$-carotene $(97 \%)$ and squalene $(97 \%)$ were obtained from Sigma-Aldrich (Darmstadt, Germany). The origin of bixin $(96 \%)$ was not specified.

\subsection{TLC Chromatography}

Concentrated aliquots from the extract solutions were applied onto silica gel 60 plates on aluminum backs (CCF-C/25, Merck, Darmstadt, Germany), which were used as the stationary phase. TLC analyses were performed with 
the aid of a glass capillary tube. Ultraviolet light (UV)-sensitive (F254) and non-UV-sensitive plates were used in this study.

TLC plates were inserted into glass chambers and subjected to elution with the mobile phase. The visualization of the bands generated by constituents that were not observed in the visible region was performed by an UV light (Multiband UV-254-366nm, UVGL-58, Mineralight ${ }^{\circledR}$ Lamp, Upland, CA, EUA) equipped with a cabinet (UVP-Chromato-VUE, CC-10, Upland, CA, EUA) with a short wavelength of $254 \mathrm{~nm}$ and a long wavelength of $366 \mathrm{~nm}$.

For compounds that did not emit fluorescence in the UV light, the visualization of the bands was performed by spraying reagent solutions (spray or visualization reagents) onto the TLC plates to color the bands of compounds. After the exposure, UV light was used to visualize the bands.

\subsection{TLC Plate Application}

\subsubsection{Mobile Phase Selection}

Initially, TLC analyses were performed by comparing three different proportions and/or combinations of solvents as shown in Table 1.

Table 1. The composition of mobile phase tested

\begin{tabular}{lcc}
\hline \multicolumn{1}{c}{ Mobile phase } & Composition (v/v/v) & Reference \\
\hline n-hexane: ethyl acetate: formic acid & $78: 18: 03$ & {$[26]$} \\
Ethyl acetate: petroleum ether & $60: 40$ & {$[27]$} \\
n-hexane: ethyl acetate & $80: 20$ & This study \\
\hline
\end{tabular}

SFE annatto extracts and the bixin standard were diluted in acetone until a final concentration $(2 \mathrm{mg} / \mathrm{ml}$ and $4 \mathrm{mg} / \mathrm{ml})$ for better resolution of TLC plates was reached. Approximately $8 \mu \mathrm{L}$ of extract solution was spotted on the plates with a capillary glass tube.

\subsubsection{Phenolic Compounds and Flavonoids}

For the visualization of flavonoids and phenolic compounds, the method of Wagner and Bladt [28] was used without applying polyethylene glycol (PEG).

A 2-aminoethyl-diphenylborinate (NP) spray reagent was prepared in a $250 \mathrm{ml}$ conical flask by diluting $1 \mathrm{~g}$ of 2-aminoethyl-diphenylborinate in $100 \mathrm{ml}$ of methanol.

After spraying the plates with the NP solution, they were visualized with UV light at a wavelength of $366 \mathrm{~nm}$.

\subsubsection{Volatile Oil Composition}

The methodology of Wagner and Bladt [28] was adopted for visualization of volatile oil constituents. A spray reagent of sulfuric anisaldehyde (SA) was prepared in a $250 \mathrm{ml}$ conical flask by diluting $0.5 \mathrm{ml}$ of p-anisaldehyde in $10 \mathrm{ml}$ of glacial acetic acid, $85 \mathrm{ml}$ of methanol and $5 \mathrm{ml}$ of concentrated sulfuric acid in this order, which formed an intense exothermic reaction.
The plates were sprayed with SA. The bands were visualized in the visible region after heating the plates in an oven (Tecnal, TE-385-1, Piracicaba, São Paulo) at 373K for 10 minutes.

\subsubsection{Antioxidant Compounds}

The revelation of antioxidant compounds was done by spraying a DPPH solution [26] in which $0.5 \mathrm{~g}$ of 2 , 2-diphenyl-1-picrylhydrazyl was diluted in $250 \mathrm{ml}$ of methanol.

The bands were visualized under natural light to verify the presence of yellow spots versus a purple background. The TLC plates were sprayed with DPPH at time zero $\left(\mathrm{t}_{0}\right)$, after 15 minutes $\left(t_{15}\right)$ and after 30 minutes $\left(t_{30}\right)$. The presence of yellow spots indicates the presence of antioxidant constituents in the sample. Bixin and 30\% Tocomin were used for the standards.

\section{Results and Discussion}

\subsection{Mobile Phase Selection and Identity of the Extracts}

The eluted TLC plates with the following mobile phases are shown in Figure 1:a) n-hexane: ethyl acetate: formic acid (78:18:3); b) ethyl acetate: petroleum ether (60:40); and c) n-hexane: ethyl acetate (80:20).

The n-hexane: ethyl acetate: formic acid (78:18:3) mobile phase achieved the best separation of bands as shown in Figure 1a.

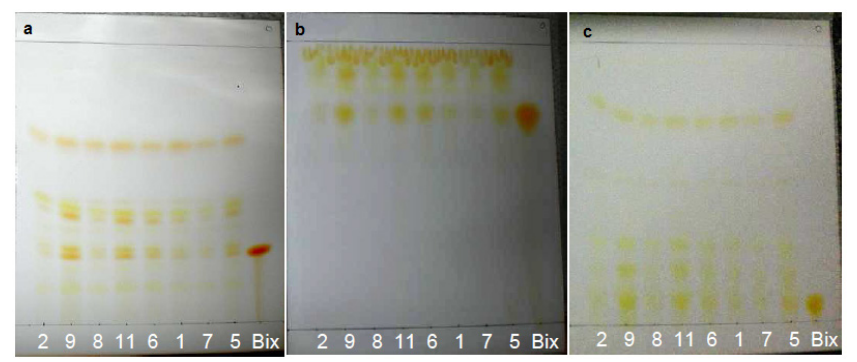

Figure 1. Selection of the optimal mobile phase of annatto extracts obtained by GYI and bixin (Bix) with the following mobile phases: n-hexane: ethyl acetate : formic acid (a), ethyl acetate : petroleum ether (b) and n-hexane : ethyl acetate (c)

The extracts from the global yield isotherms (GYIs) determined by the first batch of annatto seeds are shown in Table 2. Extracts 12,13 and 14 correspond to those obtained in each pressure profile cycle, namely pressurization, static extraction and depressurization, which were collected separately to verify their composition.

Extract 15 corresponds to the extract obtained by dynamic extraction with $\mathrm{S} / \mathrm{F}=35$, and extract 16 corresponds to the extract obtained with $\mathrm{S} / \mathrm{F}=66$. The $\mathrm{S} / \mathrm{F}$ ratio is defined as the quantity of solvent necessary to exhaust the raw material and is calculated as the ratio between the mass of solvent and the mass of material to be extracted (on a dry basis).

Table 3 shows the standards and samples used for 
identifying the SFE extract compounds.

Table 2. Identity of extracts obtained by applying GYIs to TLC plates

\begin{tabular}{ccc}
\hline Extract & Temperature (K) & Pressure (MPa) \\
\hline 1 & 313 & 20 \\
2 & & 20 \\
3 & 31 \\
4 & 333 & 31 \\
5 & & 40 \\
6 & & 40 \\
7 & 20 \\
8 & 20 \\
9 & 40 \\
10 & 40 \\
11 & 31 \\
12 & 31 \\
13 & 31 \\
14 & 31 \\
15 & 31 \\
16 & & 31 \\
\hline
\end{tabular}

Table 3. Identity of standards and extracts in the TLC plates with $8 \mathrm{~cm}$ elution height and $\mathrm{Rf}$ values

\begin{tabular}{cccc}
\hline Identity & Standard/Sample & Height $(\mathbf{c m})$ & Rf $(\mathbf{c m} / \mathbf{c m})$ \\
\hline 1 & $\beta$-sitosterol/ $\beta$-sist & 2.8 & 0.35 \\
2 & $\beta$-carotene/ $\beta$-car & 7.5 & 0.94 \\
3 & $\alpha$-tocopherol/ $\alpha-\mathrm{T} 1$ & $3.4-3.6$ & $0.43-0.45$ \\
4 & Squalene/Sq & $7.3-7.5$ & $0.91-0.94$ \\
5 & Stigmasterol/St & 2.8 & 0.35 \\
6 & Tocomin 30\%/ $\delta$-T3 & $2.6-2.7$ & $0.33-0.34$ \\
7 & SFE Extracts & & \\
\hline
\end{tabular}

The compound bands from the extracts detected in the visible region are shown in Figure 2.

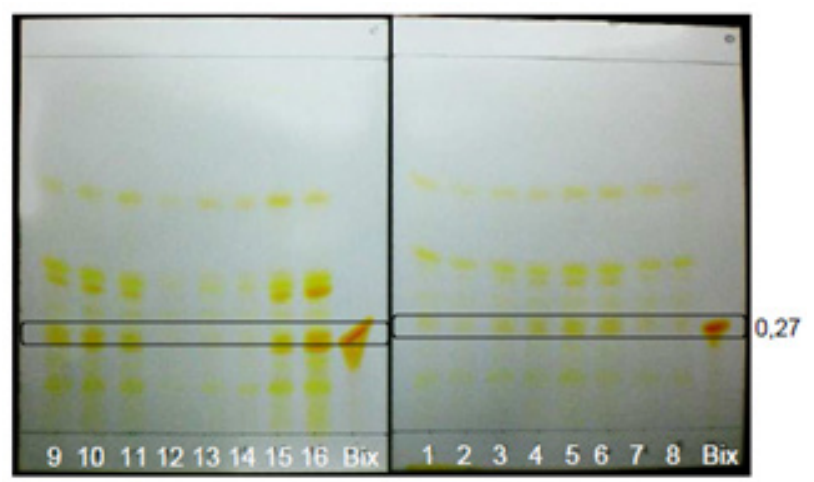

Figure 2. Extracts from GYIs. Visible zone shown without spray reagent

Bixin (Bix), as a reference standard, enabled the identification of this compound in the extracts $(\mathrm{Rf}=0.27)$. The chemical profile was similar for all the studied conditions. The most intense bands were visualized at high temperature conditions (Table 2: extracts 9-10 and 11-16), which correlated to the results published by Albuquerque and Meireles [6], who found that annatto extracts obtained at $333 \mathrm{~K} / 31 \mathrm{MPa}$ and $333 \mathrm{~K} / 40 \mathrm{MPa}$ presented the highest amounts of bixin.

Figure 3 shows the bands of compounds in the extracts visualized with UV light at $254 \mathrm{~nm}$ without spray reagent.

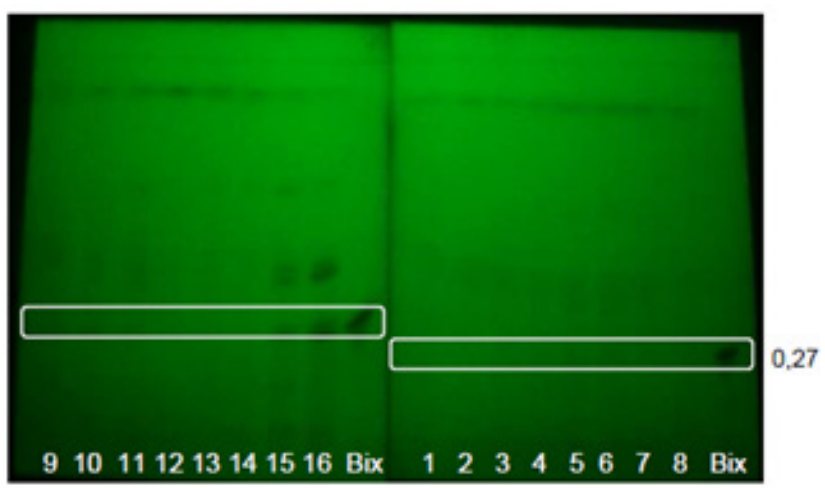

Figure 3. Extracts from GYIs. Bands visualized with UV light at $254 \mathrm{~nm}$ without spray reagent using UV-sensitive TLC plates

With the conditions used in this study, substances that possess at least two chromophores extinguish the fluorescence and appear as dark areas versus a green fluorescent light in the plates [28]. The bixin standard presented this feature, and it was found only in extracts 15-16 (Figure 3). The concentration of bixin in the other extracts may not have been sufficient to extinguish the fluorescence emitted from the TLC plate.

Figure 4 shows the compound bands in the extracts visualized with UV light at $366 \mathrm{~nm}$ without spray reagent.

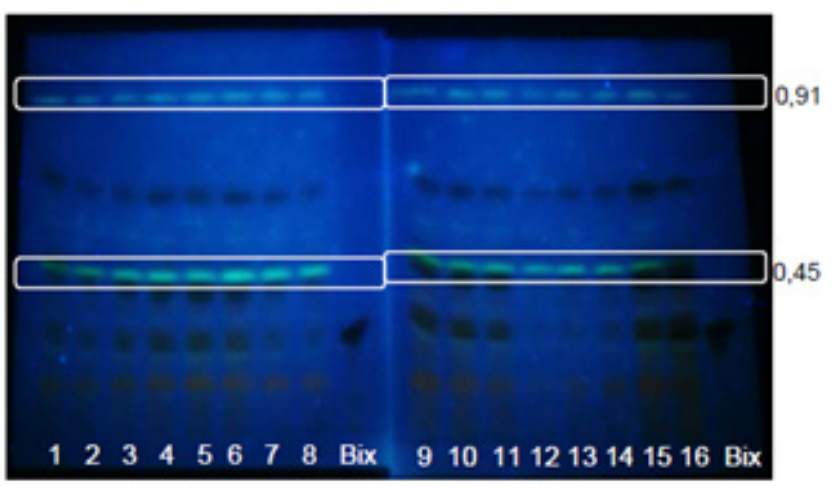

Figure 4. Extracts from GYIs. Bands visualized with UV light at $365 \mathrm{~nm}$ without spray reagent

At these conditions, terpenoids and polyphenols do not show fluorescent character. Nevertheless, fluorescent bands $(\mathrm{Rf}=0.45$ and 0.91$)$ were visualized in all the extracts, mainly in extracts 5-6, 9-10 and 12-16, which showed more intensity.

Figure 5 shows the bands of phenolic compounds visualized with a UV light at $366 \mathrm{~nm}$ without spray reagent using a UV-sensitive TLC plate. 


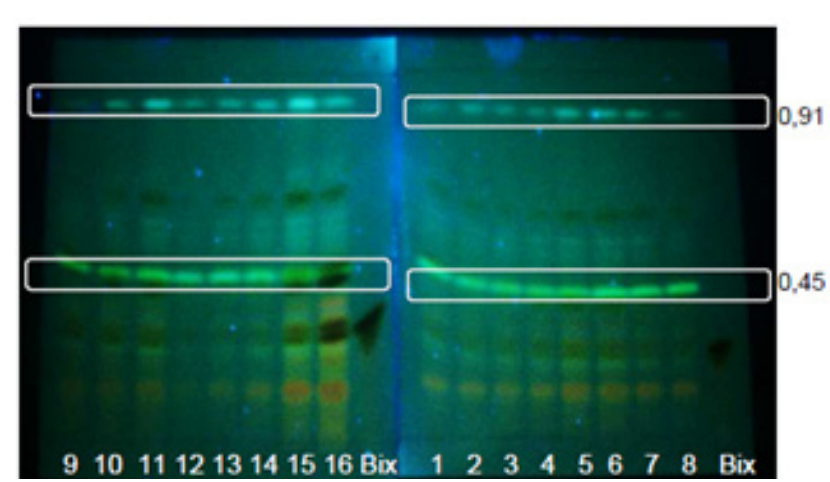

Figure 5. Extracts from GYIs. Bands visualized with UV light at $366 \mathrm{~nm}$ without spray reagent using UV-sensitive TLC plates

The fluorescent bands shown in Figure 4 were visualized with greater clarity due to the intensity in extracts $5,6,15$ and 16 (dynamic extraction obtained separately after the pressure step).

The bands of antioxidant compounds found in the extracts (Figure 6) were clearly visualized after spraying with DPPH. DPPH is a stable free radical, which can be reduced by the contact with an antioxidant substance that donor hydrogen, thereby causing the change from violet (characteristic of the radical) to yellow as the reaction proceeds. The intensity of yellow coloration is proportional to the quantity of substances with antioxidant potential [29].

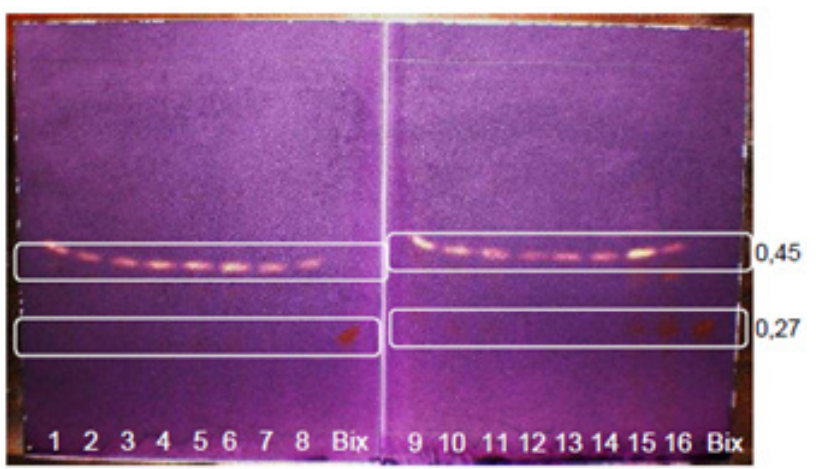

Figure 6. Extracts from GYIs. Visible region visualized with DPPH

The yellow band $(\mathrm{Rf}=0.45)$ shown in Figure 6 was found in all the extracts from all the studied conditions. The bixin standard $(\mathrm{Bix}, \mathrm{Rf}=0.27)$ bands were visualized only in extracts 15-16 and presented less intensity, thus indicating lower antioxidant activity than the compounds with $\mathrm{Rf}$ value of 0.45 .

An annatto chlorophormic extract obtained by the Soxhlet method was analyzed using the same mobile phase and DPPH, which resulted in one compound that presented the same antioxidant behavior observed in Figure 4 at $R f=0.47$ [26].

Because this technique cannot provide confirmatory results, the author confirmed the presence of tocols by high performance liquid chromatography (HPLC) with higher amounts of tocotrienol compared to the quantity of tocopherol in the studied oil.

\subsection{Phenolic Compounds and Flavonoids}

Figure 7 shows the bands of compounds after spraying with NP. Flavonoids are characterized by fluorescence observed with UV light at $365 \mathrm{~nm}$. Predominantly orange and yellow-green color indicates flavones and some types of flavonols, and dark green color indicates some types of flavonones. Phenolic carboxylic acids appear as fluorescent blue [28].

Figure 7 shows the bands of phenolic compounds visualized with UV light at $366 \mathrm{~nm}$ after spraying with NP solution.

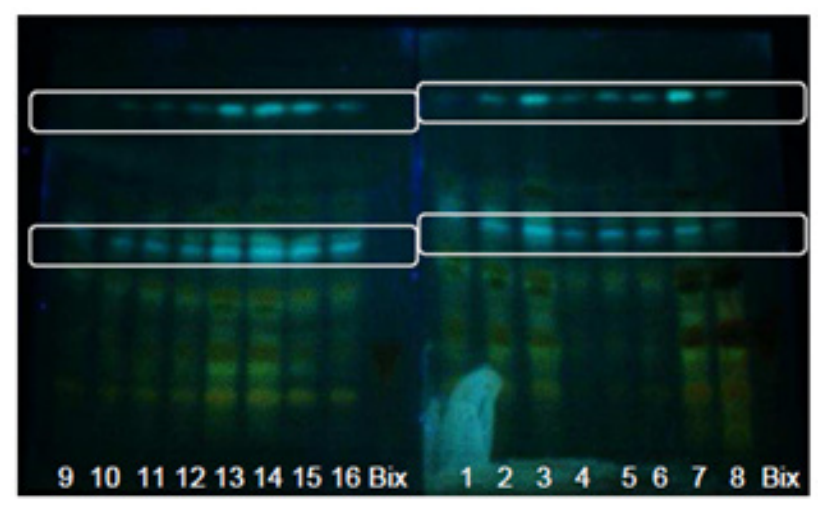

Figure 7. Extracts from GYIs. Bands visualized with UV light at $366 \mathrm{~nm}$ with NP using an UV-sensitive plate

Bands characterized by these attributes were observed (highlighted), thus indicating the presence of flavonoids and phenolic carboxylic acids in all extracts.

The color of isolated phenolic compounds from annatto seeds observed by Mittal et al. [30] was light yellow with a Rf value of 0.52 .

\subsection{Volatile Oil Composition}

Studies on the chemical composition of volatile components from water and oil-soluble annatto extracts [31] and volatile annatto oil [32] have utilized gas chromatography and mass spectroscopy to quantitatively analyze the components.

Adopting a qualitative method, a p-anisaldehyde sulfuric solution (SA) was used as a spray reagent (Figure 8) to visualize the constituents in volatile oils. This reagent also detects terpenoids, pungent principles, bitter principles and saponins.

Volatile oil compounds present dark blue, green, red and brown coloring [28]. Dark blue and brown bands were observed in the GYI extracts acquired from all the studied conditions. Nevertheless, only extracts 15 and 16 presented dark blue bands and Rf value lower than 0.5 (highlighted).

\subsection{Antioxidant Compounds}

High intensity bands for $\delta$-tocotrienol and traces of $\beta$-tocotrienol were reported on the lipidic fraction extracted from annatto seeds using n-hexane/diethyl ether as the mobile phase in the work of Frega et al. [33]. 


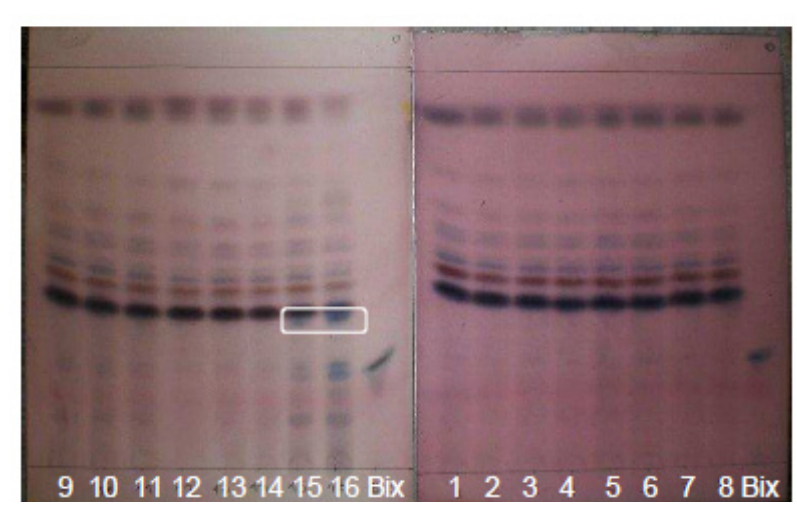

Figure 8. Extracts from GYIs. Volatile oils visualized with AS

Figure 9 shows the bands of standards, $30 \%$ Tocomin and SFE extracts, which were identified in Table 3 . The bands of carotenoids, namely $\beta$-carotene and bixin/norbixin, from samples 6 and 7, respectively, were visualized in the visible region (Figure 9a).

Constituent $\beta$-carotene was identified by comparison of its Rf (Table 3). In the UV-254 nm light (Figure 9b), compounds that extinguish the fluorescence were visualized in the $\alpha$-tocopherol standard and samples 6 and 7. According to the chemical structure of tocopherols and tocotrienols, conjugated double bonds in the cyclic chain from both compounds may have extinguished the fluorescence of the plate.

Constituents $\gamma$-T3 and $\delta$-T3 are among the tocopherols with higher $\mathrm{Rf}$ values when the normal phase (polar stationary phase: silica gel) is used in separating or detection analyses [34]. The two yellow bands from sample 6 (Figure 8 $\mathrm{c}$ and d), which were mostly retained (lower Rf value), were also observed in sample 7 (same $\mathrm{Rf}$ value), thereby indicating the presence of $\gamma$-T3 and $\delta-\mathrm{T} 3$ in these extracts considering the following relation: $\operatorname{Rf}_{\delta \mathrm{-T} 3}<\mathrm{Rf}_{\gamma-\mathrm{T} 3}$.

Figure 10 shows TLC plates used in the identification of volatile oils (Figure 10a) and antioxidant compounds (Figure $11 \mathrm{~b}$ ) in samples 6 and 7 . In sample 6 , squalene, $\beta$-sitosterol (from volatile oils), $\alpha$-tocopherol and $\beta$-carotene (antioxidants; best visualized in Figure 9b) were identified by comparison to Rf values from the respective standards. The volatile oil constituents identified in sample 6 were also found in sample 7 .

Sample 6 (30\% Tocomin) was used to identify common compounds in the SFE extracts obtained in the OEC determination at $333 \mathrm{~K}$ and $40 \mathrm{MPa}$.

In Figures 11 to 19 , extract number 1 corresponds to 5 minutes of SFE, extract number 27 corresponds to 390 minutes and extract number 28 corresponds to the extract obtained from cleaning the line out + the $\mathrm{CO}_{2}$ extract from the SFE equipment. The sample identified as " $\mathrm{T}$ " $(30 \%$ Tocomin) was used to analyze the behavior of chemical profile of extracts as a function of time.

In Figures 11-15, $\delta$-T3 shows the antioxidant activity in all annatto extracts from the OEC at zero minutes $\left(\mathrm{t}_{0}\right), 15$ minutes $\left(t_{15}\right)$ and 30 minutes $\left(t_{30}\right)$ after applying the DPPH onto plates. According to plate (a) in Figures 11 to 15, the $\delta$-T3 concentration in the extracts (1-24) decreased as a function of extraction time (5-330 minutes).

Extract number 25 resulted in an intense band due to extract 24 being collected at 320 minutes instead of 330 minutes. Extracts 25-27 showed more intense bands, which may have been due to the high concentration of $\delta$-T3 in the extract collected during the final periods of extraction.

Considering a, b and c TLC plates in Figures 11 to 16, the antioxidant activity as a function of time (after application) was detected by the increasing intensity of yellow bands in the plates not only for $\delta$-T3 but also for other constituents, which were not identified.

Figures 15 and 16 show the bands observed with visible light and UV light at $254 \mathrm{~nm}$, respectively. The bands extinguished fluorescent light had $\mathrm{Rf}$ value equal to 0.27 (equivalent value for bixin standard) and therefore were identified as bixin and/or norbixin in all OEC extracts (Figure 18).
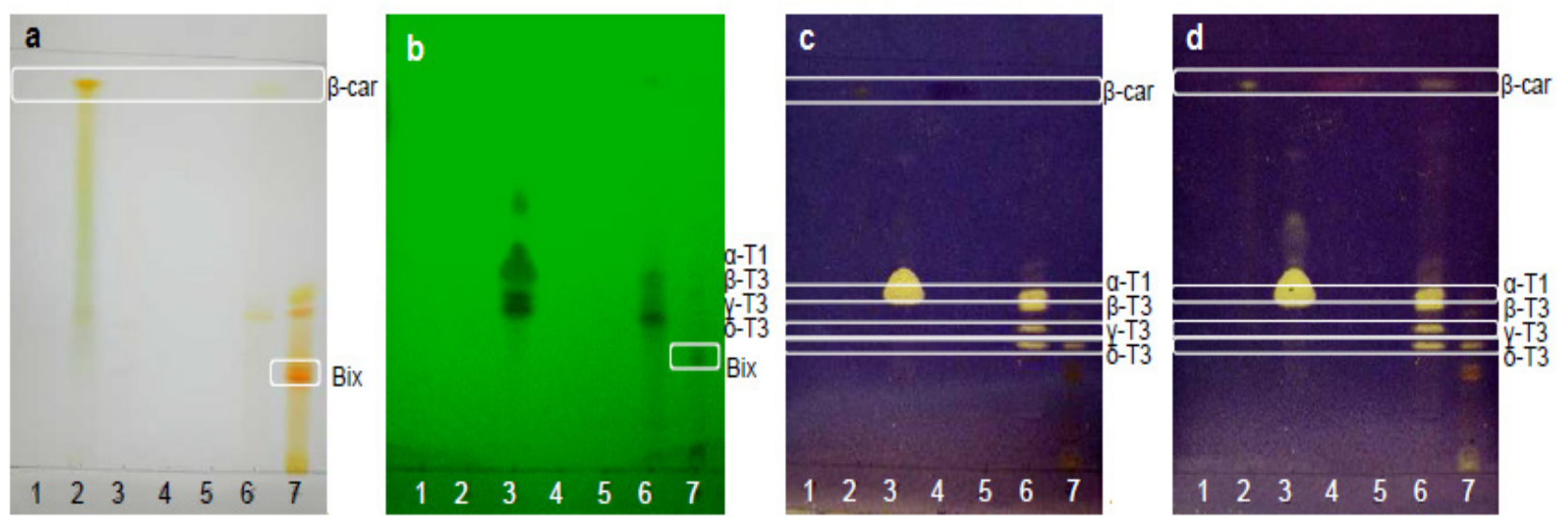

Figure 9. Standards, annatto extracts and 30\% Tocomin 30\%: (a) visible region; (b) UV-254 nm without spray reagent on UV-sensitive TLC plate; (c) visible region with DPPH in $\mathrm{t}_{0}$; and (d) visible region with DPPH at $\mathrm{t}_{30}$ 


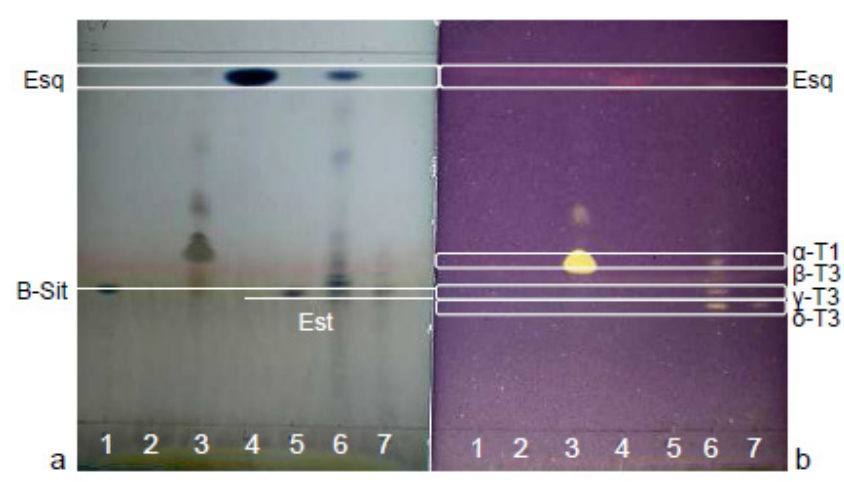

Figure 10. Standards, annatto extracts and 30\% Tocomin: (a) volatile oils in the visible region visualized with AS; and (b) antioxidant constituents in the visible zone visualized with DPPH at $\mathrm{t}_{30}$

Figure 17 shows the bands of phenolics and flavonoids in extracts from the OECs sprayed with NP using UV light at $366 \mathrm{~nm}$. The fluorescent blue bands with different Rf values were visualized in all OEC extracts. A fluorescent compound that was similar to the $30 \%$ Tocomin commercial sample was observed near the border of the solvent. Nevertheless, this compound was not identified according to the standards. Therefore, no conclusion about its identity was made.

The fluorescence intensity of the bands (Figure 17) increased in the extracts obtained in the first 50 minutes of SFE. In the final periods of SFE, the intensity of the compounds was high due to the cumulative mass of the extract.

Figure 18 shows the bands of compounds in the extracts from the OECs without spray reagent. Figure 19 shows the bands of compounds present in the extracts in the visible region after spraying with AS.

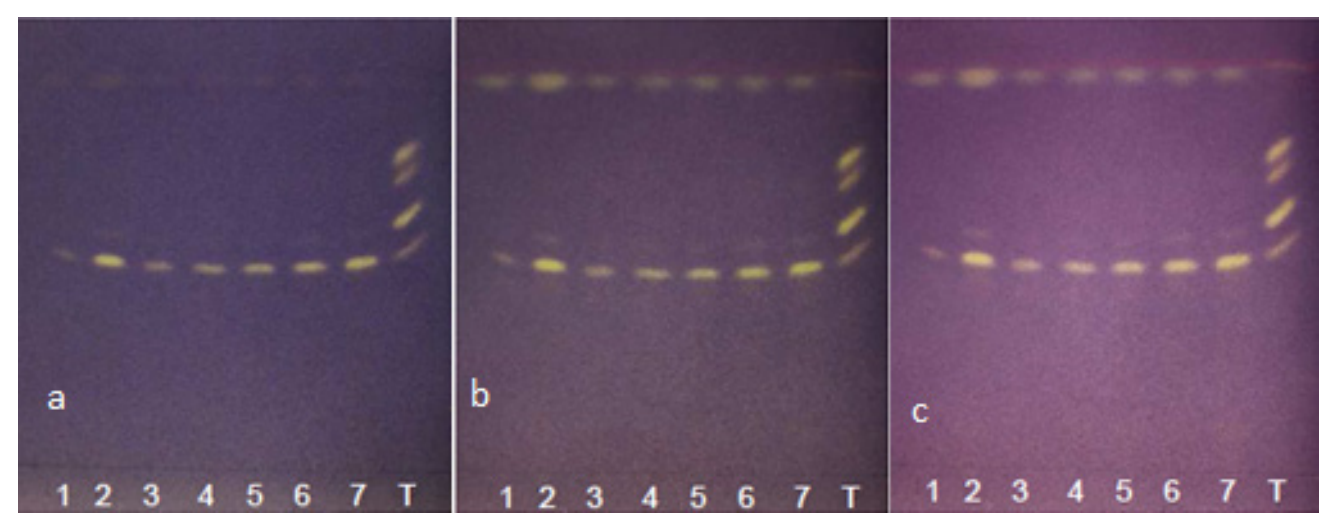

Figure 11. Annatto extracts from the OECs at 5-50 minutes in the visible zone with DPPH at (a) $t_{0}$, (b) $t_{15}$ and (c) $t_{30}$

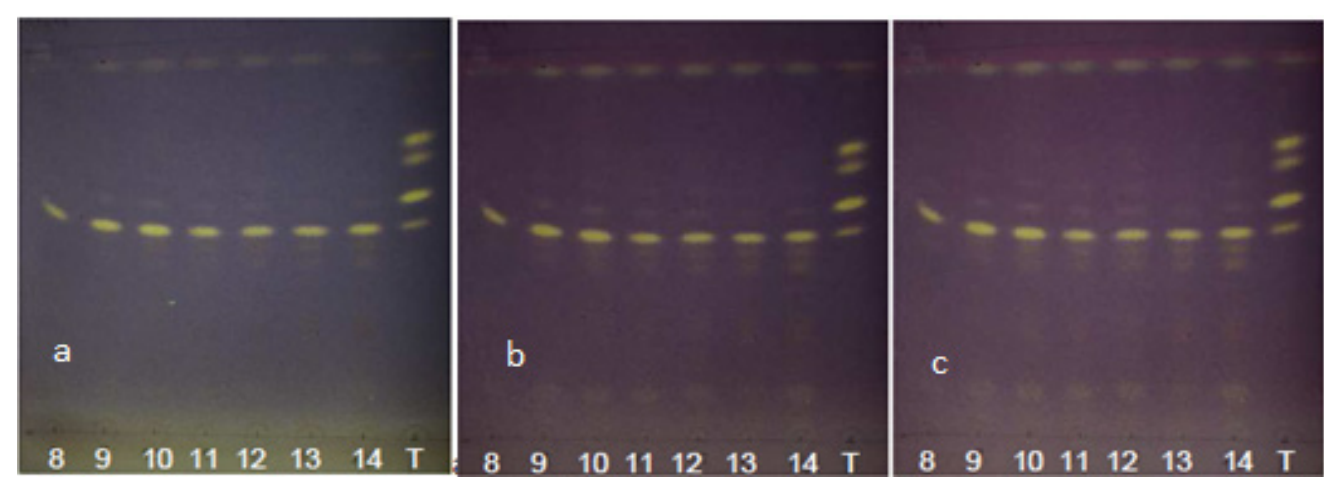

Figure 12. Annatto extracts from the OECs at 50-135 minutes in the visible region with DPPH at (a) $t_{0}$, (b) $t_{15}$ and (c) $t_{30}$

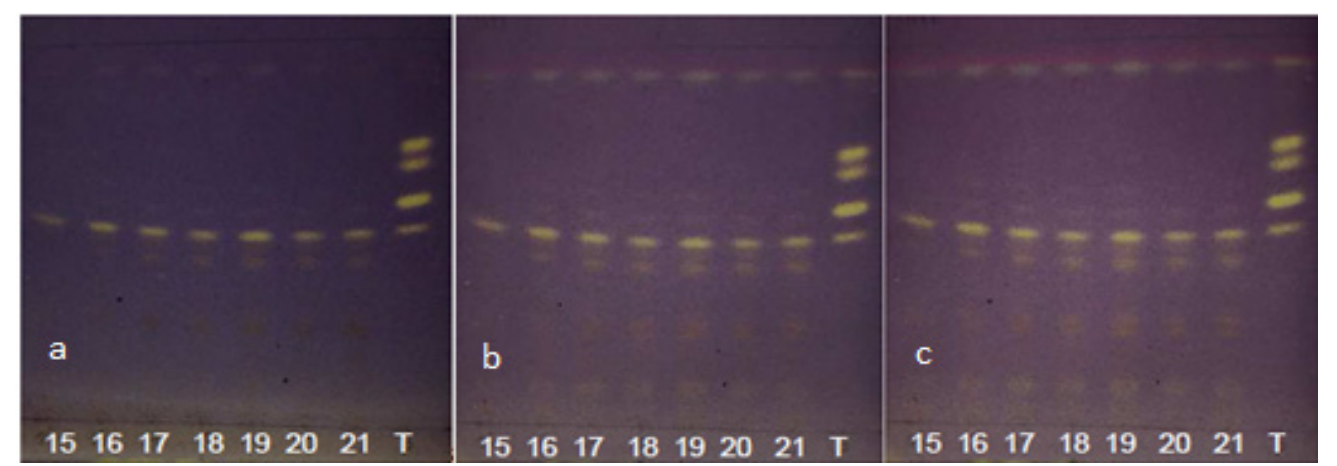

Figure 13. Annatto extracts from the OECs at 135-260 minutes in the visible region with DPPH at (a) $t_{0}$, (b) $t_{15}$ and (c) $t_{30}$ 


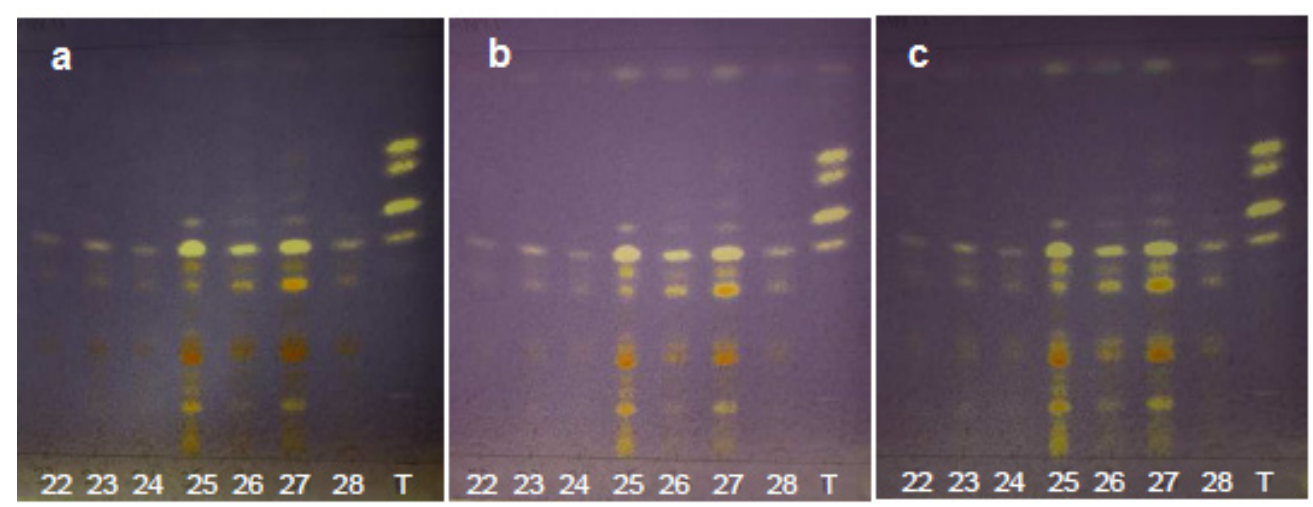

Figure 14. Annatto extracts from the OECs at 260-420 minutes in the visible region with DPPH at (a) $t_{0}$, (b) $t_{15}$ and (c) $t_{30}$
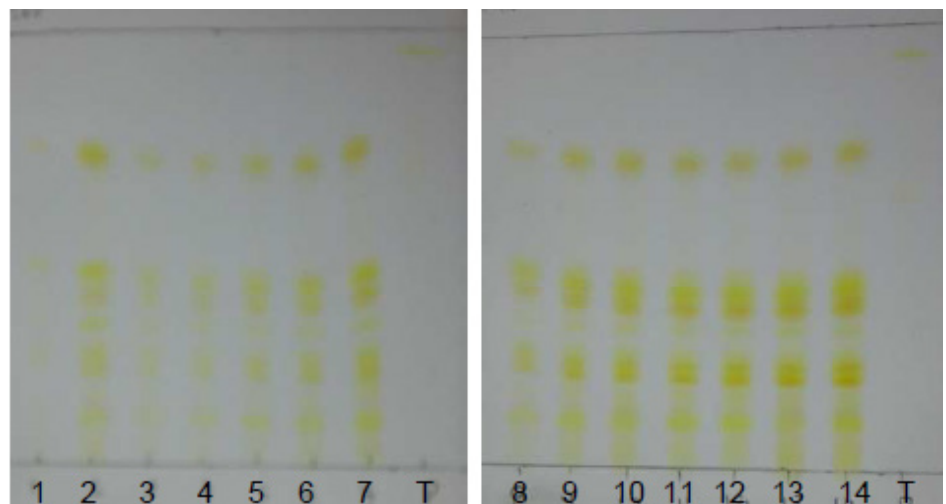

Figure 15. Annatto extracts from the OECs at 50-135 minutes in the visible region

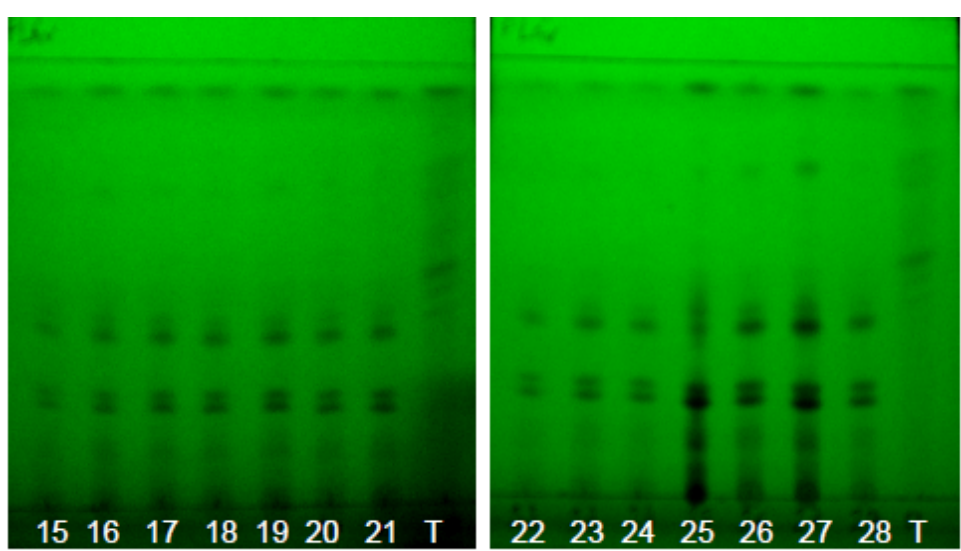

Figure 16. Annatto extracts obtained by SFE at 50-135 minutes with UV light at $254 \mathrm{~nm}$
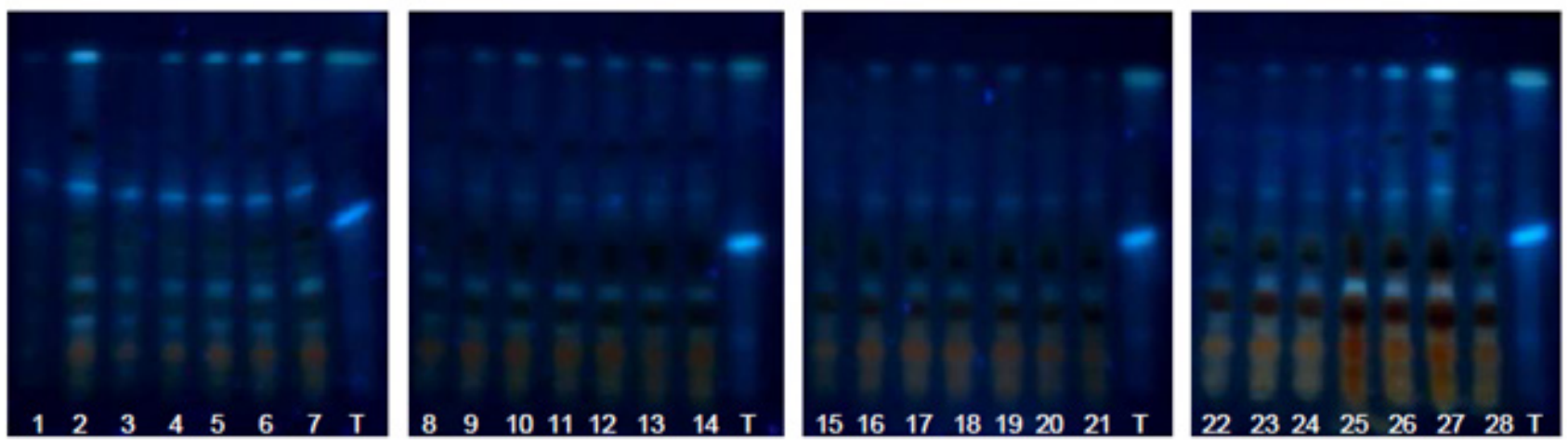

Figure 17. Annatto extracts from the OECs at 5-420 min with UV light at $366 \mathrm{~nm}$ and NP using UV-sensitive plates 

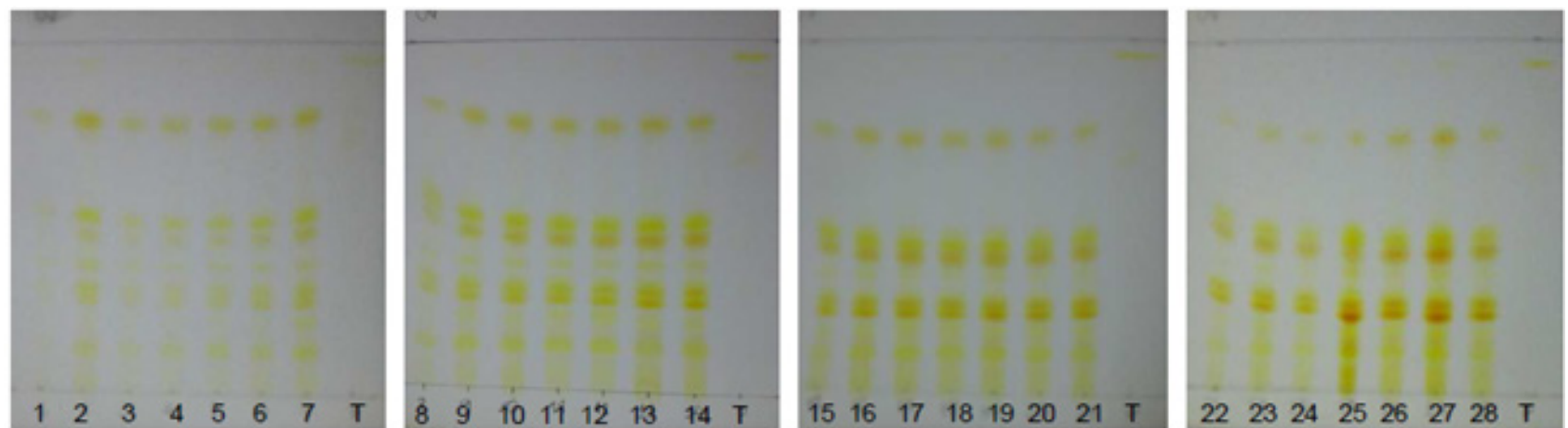

Figure 18. Annatto extracts from the OECs at 5-420 minutes in the visible region without spray reagent
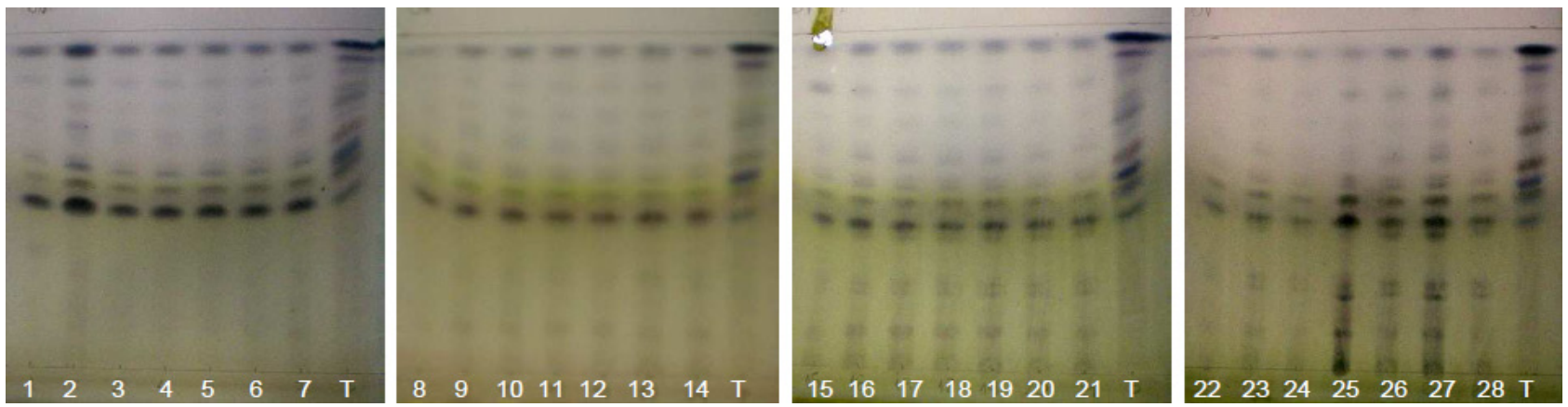

Figure 19. Annatto extract from the OECs at 5-420 minutes with volatile oils in the visible region with AS

The constituents identified in the $30 \%$ Tocomin sample were squalene $(R f=0.91-0.94)$ and $\beta$-sitosterol $(R f=0.35)$, which were also found in the SFE extracts. However, the remaining constituents were not identified.

The chemical profile of extracts varied as a function of extraction time. The intensity of antioxidants, phenolic compounds and volatile oil compounds decreased with time of extraction. However, the intensity of bixin increased with time of extraction.

The actual concentration of each compound in the extracts is unknown. However, because the intensity of the extract decreased as a function of time, the concentration of the compounds tended to increase if they were extracted at the same flow rate in a cumulative form. To quantify the compounds of interest, spectrophotometric analysis or chromatographic detectors must be employed.

\section{Conclusions}

In this study, the chemical profile of annatto extracts obtained using supercritical fluid was investigated by TLC. As a function of extraction time, the intensity of antioxidants, phenolic compounds and volatile oil compounds was reduced, while the intensity of carotenes (bixin) was increased.

Because this was a qualitative analysis, we did not evaluate the quantity or concentration, and we cannot guarantee which temperature and pressure condition provided extracts with higher or lower concentration of the studied constituents.

Based on these results, the $30 \%$ Tocomin commercial sample used for isolating the isomers of tocotrienols served as a $\gamma-\mathrm{T} 3$ and $\delta$-T3 standard for the identification analyses of antioxidants by TLC.

Although TLC is not a quantitative analysis, it is possible to select the duration of SFE process or predetermined periods for the recovery of extracts during extraction by fractionating it according to the component of interest.

\section{ACKNOWLEDGEMENTS}

Carolina L. C. Albuquerque is grateful to CNPq (Process 143092-2008-1) for her Ph.D. assistantship. Ádina L. Santana is grateful to CAPES for her Ph.D. assistantship. M. Angela A. Meireles acknowledges the productivity grant (301301/2010-7) from CNPq. The authors acknowledge financial support from $\mathrm{CNPq}$ and FAPESP (Process: 2009/17234-9). The authors are also grateful to Dr. Eliane G. Fabri (Agronomic Institute of Campinas, IAC) and Mr. W.H. Leong (Carotech Inc., Malaysia) for donating the annatto seeds and the $30 \%$ Tocomin commercial sample, respectively.

\section{REFERENCES}

[1] Mahendranath, G., Venugopalan, A., Parimalan, R., Giridhar, P., Ravishankar, G. A., 2011, "Annatto pigment production in root cultures of Achiote (Bixa orellana L.)", Plant Cell Tissue and Organ Culture, 106, (3), 517-522.

[2] Giridhar, P., Venugopalan, A., Parimalan, R., 2014, "Review 
on annatto dye extraction, analysis and processing - a food technology perspective", Journal of Scientific Research \& Reports, 3, (2), 1-22.

[3] Scotter, M., 2009, "The chemistry and analysis of annatto food colouring: a review", Food Additives \& Contaminants: Part A, 26, (8), 1123-1145.

[4] Chisté, R. C., Benassi, M. T., Mercadante, A. Z., 2011, "Effect of solvent type on the extractability of bioactive compounds, antioxidant capacity and colour properties of natural annatto extracts", International Journal of Food Science \& Technology, 46, (9), 1863-1870.

[5] Santos, L. F., Dias, V. M., Pilla, V., Andrade, A. A., Alves, L. P., Munin, E., Monteiro, V. S., Zilio, S. C., 2014, "Spectroscopic and photothermal characterization of annatto: applications in functional foods", Dyes and Pigments, 110, 72-79.

[6] Albuquerque, C. L. C., Meireles, M. A. A., 2012, "Defatting of annatto seeds using supercritical carbon dioxide as a pretreatment for the production of bixin: Experimental, modeling and economic evaluation of the process", The Journal of Supercritical Fluids, 66, 86-95.

[7] Rodrigues, L. M., Alcázar-Alay, S. C., Petenate, A. J., Meireles, M. A. A., 2014, "Bixin extraction from defatted annatto seeds", Comptes Rendus Chimie, 17, (3), 268-283.

[8] Jesus, S. P., Calheiros, M. N., Hense, H., Meireles, M. A. A., 2013, "A simplified model to describe the kinetic behavior of supercritical fluid extraction from a rice bran oil byproduct", Food and Public Health, 3, (4), 215-222.

[9] Chisté, R. C., Yamashita, F., Gozzo, F. C., Mercadante, A. Z., 2011, "Simultaneous extraction and analysis by high performance liquid chromatography coupled to diode array and mass spectrometric detectors of bixin and phenolic compounds from annatto seeds", Journal of Chromatography A, 1218, (1), 57-63.

[10] Knez, Ž., Markočič, E., Leitgeb, M., Primožič, M., Knez Hrnčič, M., Škerget, M., "Industrial applications of supercritical fluids: A review", Energy, 77, (1), 235-243.

[11] Filip, S., Vidović, S., Adamović, D., Zeković, Z., 2014, "Fractionation of non-polar compounds of basil (Ocimum basilicum L.) by supercritical fluid extraction (SFE)", The Journal of Supercritical Fluids, 86, 85-90.

[12] Griesinger, H., Fuchs, B., Süß, R., Matheis, K., Schulz, M., Schiller, J., 2014, "Stationary phase thickness determines the quality of thin-layer chromatography/matrix-assisted laser desorption and ionization mass spectra of lipids", Analytical Biochemistry, 451, 45-47.

[13] Cheng, S. C., Huang, M. Z., Shiea, J., 2011, "Thin layer chromatography/mass spectrometry", Journal of Chromatography A, 1218, (19), 2700-2711.

[14] Fried, B., Sherma, J., Thin-layer Chromatography: Technique and Applications, Vol. 35, 2nd ed., M. Dekker, Minnesota, 1986.

[15] Andrade, F. I., Guedes, M. I. F., Vieira, I. G. P., Mendes, F. N. P., Rodrigues, P. A. S., Maia, C. S. C., Avila, M. M. M., Ribeiro, L. D., 2014, "Determination of synthetic food dyes in commercial soft drinks by TLC and ion-pair HPLC", Food Chemistry, 157, 193-198.

[16] Wegener, J., Zschörnig, K., Onischke, K., Fuchs, B., Schiller,
J., Müller, K., 2013, "Conservation of honey bee (Apis mellifera) sperm phospholipids during storage in the bee queen - A TLC/MALDI-TOF MS study", Experimental Gerontology, 48, (2), 213-222.

[17] Santos, D. N., Souza, L. L., Ferreira, N. J., Oliveira, A. L., 2015, "Study of supercritical extraction from brazilian cherry seeds (Eugenia uniflora L.) with bioactive compounds", Food and Bioproducts Processing, 94, 365-374.

[18] Dias, A. M. A., Santos, P., Seabra, I. J., Júnior, R. N. C., Braga, M. E. M., Sousa, H. C., 2012, "Spilanthol from Spilanthes acmella flowers, leaves and stems obtained by selective supercritical carbon dioxide extraction", The Journal of Supercritical Fluids, 61, 62-70.

[19] Sánchez-Camargo, A. P., Martinez-Correa, H. A., Paviani, L. C., Cabral, F. A., 2011, "Supercritical CO2 extraction of lipids and astaxanthin from Brazilian redspotted shrimp waste (Farfantepenaeus paulensis)", The Journal of Supercritical Fluids, 56, (2), 164-173.

[20] Serra, A. T., Matias, A. A., Almeida, A. P. C., Bronze, M. R., Alves, P. M., de Sousa, H. C., Duarte, C. M. M., 2011, "Processing cherries (Prunus avium) using supercritical fluid technology. Part 2. Evaluation of SCF extracts as promising natural chemotherapeutical agents", The Journal of Supercritical Fluids, 55, (3), 1007-1013.

[21] Natividad, L. R., Rafael, R. R., 2014, "Carotenoid analyses and antibacterial assay of annato (Bixa orellana L.), carrot (Daucus carota L.), corn (Zea mays L.) and tomato (Solanum lycopersicum L.) extracts", Research Journal of Recent Sciences, 3, (3), 40-45.

[22] Ito, Y., Ishizuki, K., Sekigushi, W., Tada, A., Akiyama, T., Sato, K., Yamazaki, T., Akyiama, H., 2012, "Analysis of residual solvents in annatto extracts using a static headspace gas chromatography method", American Journal of Analytical Chemistry, 3, 638-645.

[23] Moraes, M. N., Zabot, G. L., Meireles, M. A. A., 2015, "Extraction of tocotrienols from annatto seeds by a pseudo continuously operated SFE process integrated with low-pressure solvent extraction for bixin production", The Journal of Supercritical Fluids, 96, 262-271.

[24] Collins, C., Braga, G. L., Bonato, P. S., Fundamentos de Cromatografia, Unicamp, Campinas, 2006.

[25] Seal, H. P., Ali, M. A., Ali, M. U., Akhter, M. H., Sultana, F., 2012, "Thin layer chromatographic analysis of food colorants from three morphotypes of annatto (Bixa orellana L.)", International Journal of Agricultural Research, Innovation and Technology, 2, (1), 7-12.

[26] Costa, C. K., "Estudo fitoquímico de Bixa orellana L. bixaceae e aplicação de seu óleo em formulação cosmética", Pharmaceutical Sciences Masters, Universidade Federal do Paraná, 2007.

[27] Tochini, L. S., Mercadante, A. Z., 2001, "Extração e deteminação, por CLAE, de bixina e norbixina em coloríficos", Ciência e Tecnologia de Alimentos, 21, 310-313.

[28] Wagner, H., Bladt, S., Plant Drug Analysis: A Thin Layer Chromatography Atlas, 2nd ed., Springer, Berlin, 2001.

[29] Hirata, L. L., "Avaliação da capacidade antioxidante de extratos de Bauhinia microstachya (Raddi) Macbride, cesalpiniaceae, em serum", Masters Thesis, Universidade 
Federal do Paraná, 2004.

[30] Mital, P., Mandev, P., Khomendra, S., Anil, B., 2013, "Antimicrobial evaluation of phenolic fraction of Bixa orellana L. bixaceae", Journal of Global Pharma Technology, $1,(5), 11-14$.

[31] Galindo-Cuspinera, V., Lubran, M. B., Rankin, S. A., 2002, "Comparison of volatile compounds in water- and oil-soluble annatto (Bixa orellana L.) extracts", Jounal of Agricultural and Food Chemistry, 50, 2010-2015.
[32] Pino, J. A., Correa, M. T., 2003, "Chemical composition of the essential oil from annatto (Bixa orellana L.) seeds", Journal of Essential Oil Research, 15, (2), 66-67.

[33] Frega, N., Mozzon, M., Bocci, F., 1998, "Identification and estimation of tocotrienols in the annatto lipid fraction by gas chromatography-mass spectrometry.", Journal American Oil Chemists' Society, 75, 1723-1727.

[34] Abidi, S. L., 2000, "Chromatographic analysis of tocol-derived lipid antioxidants", Journal of Chromatography A, 881, (1-2), 197-216. 